\title{
SOME REMARKS ON A RECENT PAPER BY BORCH *)
}

\author{
Paul Markham Kahn **) \\ Fairfax, Calif., U.S.A.
}

In his recent paper, "An Attempt to Determine the Optimum Amount of Stop Loss Reinsurance", presented to the XVIth International Congress of Actuaries, Dr. Karl Borch considers the problem of minimizing the variance of the total claims borne by the ceding insurer. Adopting this variance as a measure of risk, he considers as the most efficient reinsurance scheme that one which serves to minimize this variance. If $x$ represents the amount of total claims with distribution function $F(x)$, he considers a reinsurance scheme as a transformation of $F(x)$. Attacking his problem from a different point of view, we restate and prove it for a set of transformations apparently wider than that which he allows.

The process of reinsurance substitutes for the amount of total claims $x$ a transformed value $T x$ as the liability of the ceding insurer, and hence a reinsurance scheme may be described by the associated transformation $T$ of the random variable $x$ representing the amount of total claims, rather than by a transformation of its distribution as discussed by Borch. Let us define an admissible transformation as a Lebesgue-measurable transformation $T$ such that

$$
\begin{aligned}
& \text { (a) } 0 \leq T x \leq x \text {, and } \\
& \text { (b) } c=\int_{0}^{\infty}(x-T x) d F(x),
\end{aligned}
$$

where $c$ is a fixed number between $o$ and $m=E(x)$. Condition (a) implies that the insurer will never bear an amount greater than the actual total claims. In condition (b), $c$ represents the reinsurance premium, assumed fixed, and is equal to the expected value of the difference between the total amount of claims $x$ and the total retained amount of claims $T x$ borne by the insurer. Let us define

*) Presented to the I96I ASTis Colloquium.

* *) National Science Foundation Cooperative Fellow and Actuarial Science Fellow, The University of Michigan. 
$m^{\prime}=m-c$, and let us note then that, since $m=\int_{0}^{\infty} x d F(x)$, condition (b) could be rephrased as

$$
m^{\prime}=E(T x)=\int_{0}^{\infty} T x d F(x) .
$$

For this class of admissible transformations, we shall show that the transformation associated with stop loss reinsurance serves to minimize the variance of the cedent's liability. We shall then consider a modified set of transformations for which this result is not necessarily true, the set of $n$-transformations. We define an $n$-transformation as a Lebesgue-measurable transformation $T_{n}$ which, for fixed $n$, is such that

$$
\begin{aligned}
& \text { (a) } T_{n} x=x \text {, for } x<n \text {, and } \\
& \text { (b) } c=\int_{0}^{\infty}\left(x-T_{n} x\right) d F(x)=\int_{n}^{\infty}\left(x-T_{n} x\right) d F(x),
\end{aligned}
$$

where $c$ is, as before, the fixed reinsurance premium. Admissible transformations form a subset of the totality of all n-transformations for all $n \geq 0$; trivially, they form a subset of all o-transformations. For a fixed $n$, we shall determine that $n$-transformations which minimizes the variance of the total retained claims. Finally, we shall mention a particular kind of admissible $n$-transformation related to coinsurance.

Our problem is to determine the admissible transformation which minimizes the variance of the insurer's liability on the retained portfolio subject only to a fixed reinsurance premium $c$. As a first step in the solution, we give the following lemma.

Lemma I. There exists a unique $n_{0}$ such that $c=\int_{n_{0}}^{\infty}\left(x-n_{0}\right)$ $d F(x)$. If we let $H(n)=c-\int_{n}^{\infty}(x-n) d F(x)$, the proof of this lemma follows easily from noticing that $H(n)$ is continuous, that $H^{\prime}(n)$ is nonnegative, that $H(0)=c-m<0$ and that $H(\infty)=$ $c>0$.

Let us next define the stop loss transformation $T^{*}$ as

$$
\begin{aligned}
& T^{*} x=x, \text { for } x<n_{0} \text { and } \\
& T^{*} x=n_{0}, \text { for } x \geq n_{0},
\end{aligned}
$$


where $n_{0}$ is as in the above lemma. $T^{*}$ represents a reinsurance plan whereby the insurer pays the total claims if they are less than $n_{0}$ and pays only $n_{0}$ for total claims in excess of $n_{0}$ while the reinsurer pays the excess $x-n_{0}$.

Throughout this paper, we shall abbreviate the variance of a random variable by $V$. We then note the following lemma.

Lemma 2. $T^{*}$ is an admissible transformation and if $V^{*}=$ $V\left(T^{*} x\right)$, then $V^{*}=\int_{0}^{n_{0}}\left(x-n_{0}\right)^{2} d F(x)-\left(m^{\prime}-n_{0}\right)^{2}$. The proof of this lemma readily follows from the well-known result that, if $M$ is any number and $x$ is any random variable with mean $m$, $E(x-m)^{2}=E(x-M)^{2}-(m-M)^{2}$.

We now proceed to the principal theorem, that the transformation $T^{*}$ serves to minimize the variance of the amount of total claims on the retained portfolio.

Theorem I. If $T$ is any admissible transformation and $V^{T}=$ $V(T x)$, then $V^{*} \leq V^{T}$.

Proof: Since $T$ is admissible, $T x \leq x$. Therefore, if $x \leq n_{0}$, then $\left(T x-n_{0}\right)^{2} \geq\left(x-n_{0}\right)^{2}$. Also, since $m^{\prime}=\int_{0}^{\infty} T x d F(x)$, we have that $V^{T}=\int_{0}^{\infty}\left(T x-n_{0}\right)^{2} d F(x)-\left(m^{\prime}-n_{0}\right)^{2} \geq \int_{0}^{n_{0}}\left(T x-n_{0}\right)^{2} d F(x)-$ $\left(m^{\prime}-n_{0}\right)^{2} \geq \int_{0}^{n_{0}}\left(x-n_{0}\right)^{2} d F(x)-\left(m^{\prime}-n_{0}\right)^{2}=V^{*}$.

This proves Dr. Borch's result for the class of admissible transformations, presumably a wider class than the one he considered. It shows that stop loss reinsurance produces a smaller variance than any other type of reinsurance associated with an admissible transformation. The above proof was suggested by Professor James G. Wendel who phrased the problem in general measure-theoretic terms.

We propose now to discuss the set of $n$-transformations defined above. This is a wider set than the class of admissible transformations. We note that the stop loss transformation $T^{*}$ is an $n_{0^{-}}$ transformation; we wish to examine how its variance relates to that of an $n$-transformation with $n$ not equal to $n_{0}$. Such $n$-trans- 
formations arise in considering counter-examples to Dr. Borch's assertion, and, in fact, the stop loss transformation is no longer of minimum variance if choice from the set of $n$-transformations, $n<n_{0}$, is permitted.

An $n$-transformation represents a reinsurance plan whereby the cedent pays the full amount of all claims less than $n$ and pays $T_{n} x$ on claims greater than $n$; as before, $c$ represents the reinsurance premium. Let us note that, for $x \geq n, T_{n} x$ need not be less than $x$, the essential condition for a transformation to be admissible. Not all $n$-transformations have practical significance, but we shall investigate this set first in general and then attempt to limit the set by excluding certain transformations as impractical.

In considering these $n$-transformations, we shall make use of the function $N(n)$ defined by $N(n)=E\left(T_{n} x \mid x>n\right)$. If we denote I $-F(n)$ by $a_{n}$, we see from the following equation that $N(n)$ is independent of the particular choice of $n$-transformation $T_{n}$ and hence, for fixed $n$, is the same for all $n$-transformations.

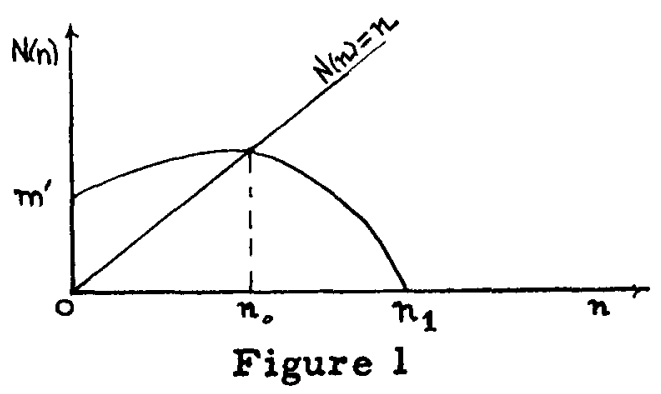

$N(n)$

$$
N(n)=E\left(T_{n} x \mid x>n\right)=\frac{\int_{n}^{\infty} T_{n} x d F(x)}{a_{n}}=\frac{\int_{n}^{\infty} x d F(x)-c}{a_{n}}
$$

In the remaining discussion, we shall assume that $F(x)$ has a density function $f(x)$. Let us note that $N(0)=m-c=m^{\prime}$, and that if $n_{1}$ is defined by $c=\int_{n_{1}}^{\infty} x d F(x)$ then $N\left(n_{1}\right)=0$ since $F\left(n_{1}\right)$ 
is different from I, for, if $F\left(n_{1}\right)$ were equal to $\mathrm{I}$, then $c=0$, thereby contradicting our assumption that $c$ is positive. Noting that $N^{\prime}(n)=-\frac{f(n) H(n)}{a_{n}}$ where $H(n)$ is defined in Lemma I, we then see easily that for $0 \leq n \leq n_{1}, N(n)$ has its maximum at $n_{0}$ and that $N\left(n_{0}\right)=n_{0}$. The graph of $N(n)$ is indicated in Figure I.

If $n=n_{1}$, then for any $n_{1}$-transformation $T_{n_{1}}$ we have that $c=\int_{n_{1}}^{\infty}\left(x-T_{n_{1}} x\right) d F(x)=c-\int_{n_{1}}^{\infty} T_{n_{1}} x d F(x)$ or that $\int_{n_{1}}^{\infty} T_{n_{1}} x d F(x)$ $=0$. For insurance with only positive risk sums as we consider here, $n_{1}$ is therefore a limiting case; if $n$ were greater than $n_{1}$, we may have $\int_{n}^{\infty} T_{n} x d F(x)<0$, a most unrealistic situation.

We wish now to consider the problem of finding, for a given $n$ and fixed $c$, that particular $n$-transformation which minimizes the variance of the total claims borne by the cedent on the retained portfolio. Let us then define the transformation $T_{n}^{*}$ by

$$
\begin{aligned}
& T_{n}^{*} x=x, \quad \text { for } x<n \text { and } \\
& T_{n}^{*} x=N(n), \text { for } x \geq n .
\end{aligned}
$$

Let us note that $N(n)>n$ for $n<n_{0}$ and $N(n)<n$ for $n>n_{0}$. We may then prove the following theorem.

Theorem 2. If $V_{n}^{*}=V\left(T_{n}^{*} x\right)$ and $V T_{n}=V\left(T_{n} x\right)$, then, for a given $n \geq 0$, we have that $V_{n}^{*} \leq V^{T_{n}}$.

Proof: $V^{T_{n}}=\int_{0}^{\infty}\left(T_{n} x\right)^{2} d F(x)-m^{\prime 2}=\int_{0}^{n} x^{2} d F(x)+$ $+a_{n}\left\{\int_{n}^{\infty}\left[T_{n} x-N(n)\right]^{2} \frac{d F(x)}{a_{n}}+N^{2}\right\}-m^{\prime 2} \geq \int_{0}^{n} x^{2} d F(x)+a_{n} N^{2}(n)-$ $-m^{\prime 2}=V_{n}^{*}$.

This theorem shows that, if we are to choose an $n$-transformation as the basis for a reinsurance scheme, $n$ and $c$ fixed, the transformation $T_{n}^{*}$ serves to minimize the variance of the retained portfolio. We have therefore solved the problem of minimizing this variance in the set of $n$-transformations by choosing a reinsurance scheme which concentrates all claims above $n$ at $N(n)$. We proceed to investigate $V_{n}^{*}$ somewhat further. 
Under the assumption that $F(x)$ and $N(n)$ are differentiable, we have that $V_{0}^{*}=0, \frac{d V_{n}^{*}}{d n}=f(n)[N(n)-n]^{2} \geq 0$ for all $n$, and hence $V_{n}^{*}$ has an inflection point at $n=n_{0}$. Therefore $V_{n}^{*}$ is a non-decreasing function of $n$; for $n>n_{0}$, i.e. $n>N(n), T_{n}^{*}$ is an admissible transformation and the minimum of $V_{n}^{*}$ occurs at $n=n_{0}$. If $n<n_{0}$, i.e. $n<N(n)$, however, then $T_{n}^{*} x$ is greater than $x$ for $n<x<N(n)$. In this case $T_{n}^{*}$ fails to be admissible; the reinsurance scheme associated with it is somewhat impractical, for it requires the cedent to bear an amount $N(n)$ exceeding the total claims if the amount of total claims is between $n$ and $N(n)$. The cedent would presumably pay the amount $x$ to the insured and the amount $N(n)-x$ to the reinsurer and in effect would pay for stability by post-payment of reinsurance. In more detail, the insurer prepays $c$ for reinsurance and pays the loss $x$ if $x<n$ for a total payment of $c+x$, which varies with $x$. In addition, if $n<x<$ $N(n)$, the insurerer pays $N(n)-x$ to the reinsurer, so that in this case a total of $c+x+(N-x)=c+N(n)$ is paid without variation, and this is true for $x>N(n)$; hence for all cases of total claims exceeding $n$, the insurer pays a total of $c+N(n)$. If $n=0$, then $N(n)=m^{\prime}$ and the insurer pays a total of $c+m^{\prime}=m$, without variation. If $n=N(n)$, i.e. $n=n_{0}$, we have the stop loss reinsurance defined by $T^{*}$.

Let us consider a special set of admissible $n$-transformations which describe a type of reinsurance with a coinsurance feature, the transformations $T_{n}^{b}, 0<b \leq \mathrm{I}$, defined by

$$
\begin{aligned}
& T_{n}^{b} x=x, \text { for } x<n \text { and } \\
& T_{n}^{b} x=b n+(1-b) x=n+(1-b)(x-n), \text { for } x \geq n,
\end{aligned}
$$

where $n$ is determined, for fixed reinsurance premium $c$, by $c=$ $\int_{0}^{\infty}\left(x-T_{n}^{b} x\right) d F(x)$. In this scheme, the reinsurer bears $b$ of the excess of claims above $n$ and the cedent retains $(\mathrm{I}-b)$ of this excess over $n$, i.e. total claims in excess of $n$ are shared in the proportion $b$ by the reinsurer and $(I-b)$ by the cedent.

The equation defining $n$ may be reduced to $c=b \int_{n}^{\infty}(x-n) d F(x)$ 
which may be written as $\frac{c}{b}+a_{n} n=\int_{n}^{\infty} x d F(x)$ or $N(n)-n=\frac{(\mathrm{I}-b) c}{a_{n} b}$. These equations define $b$ as a function $b(n)$ of $n$. Since $T_{n}^{b}$ is an admissible transformation, we may apply Theorem I to find that, if $V_{n}^{b}=V\left(T_{n}^{b} x\right)$, then $V_{n}^{b}$ is minimized at $n=n_{0}, b\left(n_{0}\right)=\mathrm{I}$, for these values of $n$ and $b$ clearly give the stop loss transformation $T^{*}$.

Although the variance is minimized at $b=\mathbf{I}$, an insurer or reinsurer may still prefer a scheme where $b<\mathrm{r}$, for underwriting reasons, for example. Let us note that $b(0)=\frac{c}{m}$ and $V_{n}^{b(0)}=$ $\left(\mathrm{I}-\frac{c}{m}\right)^{2} V(x)$. It is also easily seen that $\frac{d b(n)}{d n}=\frac{a_{n} b^{2}}{c}$, and as $n$ increases from o to $n_{0}, b(n)$ increases from $\frac{c}{m}$ to $\mathrm{I}$. From this it may be noted that values of $n$ larger than $n_{0}$ are impractical, for they would imply values of $b$ greater than $\mathrm{I}$, and hence, for sufficiently large claims, $T_{n}^{b} x$ would be negative.

Let us define the function $y_{x}$ of the random variable $x$ by

$$
y_{x}=x \text {, for } x \geq n
$$

and let us note that it has distribution function $\left[F_{(y)}-\mathrm{F}_{(n)}\right] / a_{n}$. Assuming that $V_{n}^{b(n)}$ is a differentiable function of $n$, we find that

$$
\frac{d V_{n}^{b}}{d n}=-\frac{2\left(a_{n} b\right)^{2}(\mathrm{I}-b)}{c} V\left(y_{x}\right)
$$

Since $b(n)>\mathrm{I}$ for $n>n_{0}$ and $b(n)<\mathrm{I}$ for $n<n_{0}$, we have $\frac{d V_{n}^{b}}{d n} \leq 0$ for $n<n_{0}$ and $\frac{d V_{n}^{b}}{d n} \geq 0$ for $n>n_{0}$. If $n=n_{0}$, then $b\left(n_{0}\right)=I$ and $\frac{d V_{n}^{b}}{d n}=o$; this confirms our finding that $V_{n}^{b}$ is minimized at $n=n_{0}$. Hence as $n$ increases from o to $n_{0}, V_{n}^{b}$ decreases from $\left(\mathrm{I}-\frac{c}{m}\right)^{2} V(x)$ to $V^{*}$.

Coinsurance is an important element of reinsurance, and for this reason we have chosen to investigate it in detail. For coinsurance 
with $b<\mathrm{I}$, the insurer's variance is somewhat greater than it is under stop loss reinsurance, and the relations obtained here provide some indication of the increase in the insurer's variance for various degrees of coinsurance.

We briefly discuss an alternate criterion to that involving the variance. Let $c$ again be a fixed positive reinsurance premium and let $T$ be a continuous, nondecreasing, admissible transformation.

Let $M=\inf \left\{x \mid T_{x}=m^{\prime}\right\}$, where $m^{\prime}=\int_{0}^{\infty} T x d F(x)$. Define the gain $G(x)$ and the loss $L(x)$ by

$$
\begin{aligned}
G(x) & =m^{\prime}-T x, L(x) & =0, \text { for } x<M, \text { i.e. } T x<m^{\prime} \\
G(x) & =0, \quad L(x) & =T x-m^{\prime}, \text { for } x \geq M .
\end{aligned}
$$

Let $R=R(T)=E\left(G^{2}\right)-E\left(L^{2}\right)$, let us choose $R$ as the basis for our new criterion, the larger $R$, the better the reinsurance scheme $T ; R$ gives some measure of how favorable the scheme is to the insurer. Preliminary study indicates that there may not be any simple solution to finding a transformation $T$ maximizing $R$. In certain special cases of practical interest, we can, however, find a solution. We shall assume that $m^{\prime} \leq n$, a not very restrictive condition, since one is usually interested in reinsuring only claims above a certain limit, usually beyond the mean $m$, and hence beyond $m^{\prime}$. This condition implies that $M=m^{\prime}$, if we consider the coinsurance transformation $T_{n}^{b}$.

We find that $R=2 \int_{0}^{M}\left(T x-m^{\prime}\right)^{2} d F(x)-V^{T}$, in general. For $T=T_{n}^{b}$, this reduces to $R=2 \int_{0}^{m^{\prime}}\left(x-m^{\prime}\right)^{2} d F(x)-V_{n}^{b}$, the first term of which is independent of $T$. Therefore, to maximize $R$, we must minimize $V_{n}^{b}$; but this problem was solved above by taking $n=n_{0}$ and $b\left(n_{0}\right)=\mathrm{I}$, i.e. for $T_{n}^{b}$ the stop loss transtormation $T^{*}$. This argument has been stated in terms of the ccinsurance transformation because it is a practical illustration of a continuous, nondecreasing, admissible $\mathbf{n}$-transformation. One may assert, however, that with choice permitted from the set of all such transformations with $m^{\prime} \leq n$, the stop loss transformation gives maximal $R$. 